\title{
Minimal repair and replacement policy for a complex system with different failure modes of the groups
}

\author{
Zaharaddeen Haruna Aliyu ${ }^{1 *}$, Bashir Maifada Yakasai Phd ${ }^{2}$ \\ ${ }^{1}$ Department of Mathematics/Computer Sciences Nigeriandefence Academy Kaduna, Nigeria \\ ${ }^{2}$ Department of Mathematical Sciences Bayero University Kano, Nigeria \\ *Corresponding author E-mail: zaharuna@nda.edu.ng
}

Copyright @ 2014 Zaharaddeen Haruna Aliyu, Bashir Maifada Yakasai Phd. This is an open access article distributed under the Creative Commons Attribution License, which permits unrestricted use, distribution, and reproduction in any medium, provided the original work is properly cited.

\begin{abstract}
This study or research work considered a complex system whose components are grouped into two each with different failure type. The first group consists of components or devices that are repairable and replaceable at failure (type y) and the second group consist of components that are non-repairable but replaceable at failure (type z). We rectify the defect of first group using minimal repair with probability $\mathrm{p}$ and replacement with probability $1-\mathrm{p}$ at some threshold number $\mathrm{n}+1, \mathrm{n}>1$ of the minimal repair, whereas for components at second group we adopt only replacement in order to correct the failure of the components. Cost functions of the model are derived and both analytical and empirical realizations are made.
\end{abstract}

Keywords: Repairable, Replaceable, Replacement and Minimal Repair.

\section{Introduction}

It is very important to avoid failure of a system during actual operation when such an event is costly and/or dangerous. In such situations, one important or relevant area of interest in reliability theory is to study various methods of maintenance policies in order to reduce the occurrence of such system failure.

Due to system failure during operation nowadays, most of the industries in the modern world spend $50-60 \%$ of their budget in maintaining their machines or systems. Therefore it is significant to implement a policy in order to reduce pressure face by those industries. In academics, we study the maintenance in order to give our contribution to those types of industries to convert the maintenance activities from necessary evil to a profit contribution by minimizing the cost of the maintenance, which in turn reduce the cost of production thereby maximizing the profit and improve the reliability of the system. Therefore in this research work, we determine the optimum maintenance cost of the complex system with two types of failure modes

The maintenance activities that include the idea of minimal repair and replacement are introduced by many mathematicians in their papers such as Sheu S.H. and Chien Y.H. [4] where they considered a generalized age replacement policy of a system subject to shocks. If the system suffers shocks and fails at age $y(\leq t)$, it is either replace by new system (if the failure is catastrophic) or it undergoes minimal repair (minor failure), otherwise the system is replaced when the first shock after the time $t$ arrives or the total operating time reaches age $T(0 \leq t \leq T)$ whichever comes first. These two possible actions during the period $[0, t]$ depends on some random mechanism which depends on the number of shocks suffered since the last replacement. This model is generalized by Chien Y.H., et al [1]. Also Sheu S.H., Griffith W.S. and Nakagawa T. [2] proposed a generalized replacement policy where a system has two types of failure and is replaced at the $\mathrm{n}$-th type I failure (minor failure) or at first type II failure (catastrophic) or at age T, whichever occurs first. Aven T. and Castro I.T. [3] consider a minimal repair and replacement model with two types of failure and a safety constraint. If the system fails, and the failure is of type I, then the system is minimally repaired. If the failure is of type II, the system is minimally repaired with probability $\mathrm{p}$ and replaced with probability 1-p. failure of type II is safety critical and to control the risk, management has specified a requirement that the probability of at least one such failure occurring in the interval $[0, \mathrm{~A}]$ should not exceed a certain probability limit $\omega$. Wang [7] also considers minimal repair, and many others such as Nakagawa [8], Wang [7], Yakasai [5]. 
In this study, we consider a complex system or machine in which it consist of different components or devices ( multi component system) where we group the components/devices into two major categories or groups each with different failure mode.

The groups are as follows

1) Group A: this group consists of the system components that are repairable and replaceable at their failure.

2) Group B: this group consists of the system components that are non-repairable but replaceable at their failure or even if they can be repaired, the probability of repairing them is very negligible as well as the reliability of the component after the repair will be very low or the cost of repair is too large.

Two types of failure of the groups are define as type y failure to be the failure of group A components which is rectifiable by minimal repair or replacement and type $\mathrm{z}$ failure to be the failure of group $\mathrm{B}$ components that is correctable only by replacement.

For group a components, we adopt minimal repair in order to rectify the defects of those components at failed condition. Minimal repair is considered to bring a component back to its condition just before the failure occur and ready for operation such that the system can sustain to its planned replacement period T. Replacement is considered for group A component at some threshold number $n+1, n>1$ of the minimal repair where the number of minimal repair has exhausted the reliability of the system, therefore it is better to replace the component than to conduct a minimal repair.

For group B components, at failure we remove and replace the failed component by new since its components failure is somehow considered to be catastrophic.

This kind of problem is commonly found in automobiles such as car, trucks, motorcycle, aero planes and others. For example, a car components can be group/classify into the above two groups, firstly, engine, gear box, carburetor and some others as group A components in which we can minimally repair at their failure, but at some threshold number $\mathrm{n}+1, \mathrm{n}>1$ of minimal repair we just adopt replacement due to high rise of the cost of repair and low reliability of the components after undergoing several number of repairs and secondly, chassis, light bulb, tires and others as group B components which they are non-repairable but replaceable or even if they can be repaired but there will be high risk or the component will have very low reliability.

In the second section we give the policy and derive the cost function, in the third section we give the optimization of the problem, numerical example is given in the fourth section and conclusion is given in the last section.

\subsection{Assumptions}

We assume the following throughout this paper

1) The groups/components of the system works dependently,

2) Failure of one component disturbs the system but does not affect the other components,

3) Components of the system have an increasing failure rate (IFR),

4) Spare parts are unlimited and always available,

5) Minimal repairs and replacement are always perfect,

6) The system failure is self-announce, that is, even if no inspection is scheduled as soon as failure occurs it will be detected, and

7) Time constraint does not affect the minimal repair or replacement.

\subsection{Notations}

Here are some notations used in this research work,

$x$, Period before failure of the system occurs,

$F(t), f(t), \mathrm{CDF}$ and PDF of the component respectively,

$r(t)$, Failure rate function of the components,

$\bar{F}(t)$, survival function of a component at time $\mathrm{t}$,

$\lambda_{1}, \lambda_{2}$, finite mean for group A and B respectively,

$R_{i}(t)$, The reliability of a component $\mathrm{i}$

$H_{o}(t)$ and $H_{m}(t)$ Ordinary and Modified renewal functions respectively,

$I_{\{.\}}$, Indicator probability function equals to 1 if the argument is true and 0 if otherwise,

$X_{T R A}$, time of replacement of component of group A type,

$\mathrm{n}$, Threshold number of the minimal repair,

$\mathrm{T}$, the time of planned replacement of the system,

, discounting factor of the cost of minimal repair and $\alpha$

$\mathrm{N}$, the counting process of the failure

Others are defined where appropriate. 


\subsection{Policy}

Before the system or machine start to operate, i.e. during and after burnt in period (where failures are not considered at the period), and letting the random variable $x$ be the working period before failures occur, we assume $f_{A}(t)$ and $f_{B}(t)$ to be the failure probability density functions $(p d f)$ for group $\mathrm{A}$ and B with finite mean $\lambda_{1}=E_{A}(t)$ and $\lambda_{2}=E_{B}(t)$ respectively. When the machine is switch to operation, the components of the machine are subject to failure, thus we can minimally repair for components of group A type with probability $p$ where $0 \leq P \leq 1$ and switch it back to operation with new failure probability density function as $f^{\prime}{ }_{A}(t)$.Thus, the process of brake/fail and minimally repair is continue up to a threshold number $n>1$ where for $n+1$ number of the minimal repair we adopt replacement with probability $1-p$, this process is simply a modified renewal process (MRP). For components of group B type, at their failure we just remove the failed component and replace it by new with the same failure probability density function, thus, this process is simply an ordinary renewal process (ORP).

\section{Cost functions derivation}

Minimal repair cost: we minimally repair the failed group A component up to threshold number $n>1$, therefore the expected cost function of the minimal repair is assumed to be

$n E$ (cost of min.rep.) $H_{m}(t)$

Where $H_{m}(t)$ is the renewal function for a modified renewal process?

Minimal repair cost includes the removal and installation costs of a component.

Replacement cost: we replace the failed group a component in three ways, and then the associated costs are:

$Y=\left\{\begin{array}{rr}C_{1 r}, & \text { if } n+1 \text { failure occur } \\ C_{p}, & \text { if age } T \text { is attained } \\ C_{c}, & \text { if first failure is catastrophic }\end{array}\right.$

Therefore the replacement costs functions for group a components are

$\left\{\begin{array}{c}(1-p) C_{1 r} H_{m}(t) \\ C_{p} H_{o}(t) \\ C_{c} H_{o}(t)\end{array}\right.$

Where $H_{o}(t)$ is the renewal function for ordinary renewal process?

For group $\mathrm{B}$, the failed component is replaced by new and the associated cost function is

$C_{B} H_{O}(t)$

Therefore, for group A, the expected costs are

$n E$ (cost of min.rep.) $H_{m}(t)+(1-p) C_{1 r} H_{M}(t)$

$n E$ (cost of min. rep.) $H_{m}(t)+C_{P} H_{o}(t)$

$n E$ (cost of min. rep.) $H_{m}(t)+C_{c} H_{o}(t)$

The total expected costs of the system are

$n E$ (cost of min.rep.) $H_{m}(t)+(1-p) C_{1 r} H_{M}(t)+C_{B} H_{O}(t)$

$n E$ (cost of min.rep.) $H_{m}(t)+C_{p} H_{o}(t)+C_{B} H_{O}(t)$

$n E$ (cost of min. rep.) $H_{m}(t)+C_{c} H_{o}(t)+C_{B} H_{O}(t)$

The total expected costs per unit time of the system are:

$C_{1}(t)=\frac{n E(\text { cost of } \min . r e p .) H_{m}(t)+(1-p) C_{1 r} H_{M}(t)+C_{B} H_{O}(t)}{t}$

$C_{2}(t)=\frac{n E(\text { cost of min.rep. }) H_{m}(t)+C_{p} H_{O}(t)+C_{B} H_{O}(t)}{t}$

$C_{3}(t)=\frac{n E(\text { cost of min.rep. }) H_{m}^{t}(t)+C_{c} H_{O}(t)+C_{B} H_{O}(t)}{t}$

We next find the expected discounted cost of the minimal repair,

The discounted cost associated with minimal repair caused by type y failure before $n+1$ failure of the system according to Aven and Castro [3] is given by

$E\left(\int_{0}^{X_{T R}} \sum_{i=1}^{n} C_{1 i m} e^{-\propto t} d N_{1, m}(t)\right)$

Where $\left\{N_{1 m}(t), t>0\right\}$ is the counting process for the minimal repair?

And since it is a non-homogeneous Poisson process with intensity function $p r_{1}(t)$, then

$E\left(\int_{0}^{X_{T R}} \sum_{i=1}^{n} c_{1 i m} e^{-\propto t} d N_{1, m}(t)\right)=E\left(\int_{O}^{\infty} \sum_{i=1}^{n} c_{1 i m} e^{-\propto t} I_{t \leq X_{T R}} d N_{1, m}\right)$

$=P \sum_{i=1}^{n} c_{1 i m} \int_{0}^{T} e^{-\propto t} \bar{F}_{T R}(t) r_{1}(t) d t$

The renewal or integral equations for modified and ordinary renewal processes according to renewal theory are respectively given as follows

$H_{m}(t)=\int_{0}^{t}\left[1+h_{m}(t-x)\right] d F_{A}(x)$

And

$H_{O}(t)=\int_{0}^{t}\left[1+h_{o}(t-x)\right] d F_{B}(x)$ 
Therefore the total average costs or cost functions from (10), (11), (12), (13) and (14) are given as:-

$$
\begin{aligned}
C_{1}(t) & =\frac{n p \sum_{i=1}^{n} C_{1 i m} \int_{0}^{T} e^{-\propto t} \overline{\mathrm{F}}_{\mathrm{TRA}}(t) \mathrm{r}_{1}(\mathrm{t}) H_{m}(t) d t+(1-p) C_{1 r} H_{m}(t)+C_{B} H_{O}(t)}{t} \\
C_{2}(t) & =\frac{n p \sum_{i=1}^{n} C_{1 i m} \int_{0}^{T} e^{-\propto t} \overline{\mathrm{F}}_{\mathrm{TRA}}(t) \mathrm{r}_{1}(\mathrm{t}) H_{m}(t) d t+C_{p} H_{m}(t)+C_{B} H_{O}(t)}{t} \\
C_{3}(t) & =\frac{n p \sum_{i=1}^{n} C_{1 i m} \int_{0}^{T} e^{-\propto t} \overline{\mathrm{F}}_{\mathrm{TRA}}(t) \mathrm{r}_{1}(\mathrm{t}) H_{m}(t) d t+C_{C} H_{O}(t)+C_{B} H_{O}(t)}{t}
\end{aligned}
$$

\subsection{Problem optimization}

We optimizes by differentiating the various cost functions to find the minimum cost. Classifying the problem into three and using exponential to make the analytical realization we have

Case 1:-when the cost function is (15)

$\frac{d}{d t} c_{1}(t)=\frac{n p \sum_{i=1}^{n} c_{1 i m} b(t) y_{m}+\rho t n p \sum_{i=1}^{n} c_{1 i m} x_{m} e^{-(\alpha+\rho) T}+(1-P) C_{1 r} y_{m}+c_{B} y_{O}}{t}$

And

$T_{\text {opt }}=\frac{-1}{\alpha+\rho} \ln \left(\frac{-\left(\frac{\rho n p}{\alpha+\rho} \sum_{i=1}^{n} c_{1 i m} y_{m}+(1-p) c_{1 r} y_{m}+c_{B} y_{o}\right)}{\rho n p \sum_{i=1}^{n} c_{1 i m}\left(t x_{m}-\frac{1}{\alpha+\rho} y_{m}\right)}\right)$

Where

Case 2:- when the cost function is (16)

$\frac{d}{d t} C_{2}(t)=\frac{n p \sum_{i=1}^{n} c_{1 i m} b(t) y_{m}+\rho t n p \sum_{i=1}^{n} c_{1 i m} x_{m} e^{-(\alpha+\rho) T}+C_{P} y_{o}+c_{B} y_{O}}{t}$

Which shows that $\frac{d}{d t} c_{2}(t)>0$ is an increasing function so that minimum of $\mathrm{C}_{2}(\mathrm{t})$ occurs at $\mathrm{T}=\infty$ and since $\frac{d^{2}}{d t^{2}} C_{2}(t)>$ 0 then it is of advantage to plan for replacement, for the distribution is exponential.

And

$T_{\text {opt }}=-\frac{1}{\alpha+\rho} \ln \left(\frac{-\left(\frac{\rho n p}{\alpha+\rho} \sum_{i=1}^{n} c_{1 i m} y_{m}+c_{p} y_{o}+c_{B} y_{o}\right)}{\rho n p \sum_{i=1}^{n} c_{1 i m}\left(t x_{m}-1 / \alpha+\rho y_{m}\right)}\right)$

Case 3:- when the cost function is (17)

$\frac{d}{d t} C_{3}(t)=\frac{n p \sum_{i=1}^{n} c_{1 i m} b(t) y_{m}+\rho t n p \sum_{i=1}^{n} c_{1 i m} x_{m} e^{-(\alpha+\rho) T}+c_{c} y_{o}+c_{B} y_{o}}{t}$

Which shows that $\frac{d}{d t} c_{3}(t)>0$ is an increasing function which shows that minimum of $\mathrm{C}_{3}(\mathrm{t})$ occurs at $\mathrm{T}=\infty$, and since $\frac{d^{2}}{d t^{2}} C_{3}(t)>0$ then it is of advantage to plan for replacement if the distribution is exponential.

And

$T_{o p t}=-\frac{1}{\alpha+\rho} \ln \left(\frac{-\frac{\rho n p}{\alpha+\rho} \sum_{i=1}^{n} c_{1 i m} y_{m}-c_{c} y_{o}-C_{B} y_{o}}{\rho n p \sum_{i=1}^{n} c_{1 i m}\left(t x_{m}-\frac{1}{\alpha+\rho} y_{m}\right)}\right)$

\subsection{NUMERICAL EXAMPLE}

We assume that the problem follows an exponential distribution and its $p d f$ is given by

$f(t)=\rho e^{-\rho t}, \quad \rho>0$

We also take into account that the $p d f$ of the system or machine components does not change even if it undergoes minimal repair. We give values to the following parameters

$c_{11 \mathrm{~m}}=2.5, c_{12 \mathrm{~m}}=2.8, c_{13 \mathrm{~m}}=3.1, c_{14 \mathrm{~m}}=3.4, c_{15 \mathrm{~m}}=3.7$

$c_{1 r}=10.6, c_{p}=9.5, c_{c}=15.5, c_{B}=11.5, \alpha=0.02, \rho=0.1$

Therefore the $p d f$ has now becomes

$f(t)=0.1 e^{-0,1 t}$

Now,

$H_{m}(t)=E(t)=\int_{0}^{t} s f(s) d s=-t e^{-0.1 t}-(1 / 0.1) e^{-0.1 t}+1 / 0.1$

$h_{m}(t)=\frac{d}{d t}\left(H_{m}(t)\right)=0.1 t e^{-0.1 t}$

Since the $p d f$ is not changing then,

$H_{m}(t)=H_{o}(t), h_{m}(t)=h_{o}(t)$

Using the above equations, the following tables are obtain

Case 1: Cost Function Table

\begin{tabular}{cccccccc}
\hline $\mathrm{n}$ & $\mathrm{P}$ & $\mathrm{t}$ & $H_{m}(t)=H_{o}(t)$ & $h_{m}(t)=h_{o}(t)$ & $Z_{m}(t)$ & $T_{O P T}=|T|$ & $C_{1}(\mathrm{~T})$ \\
\hline 1 & 0.9 & 2 & 0.1730 & 0.1640 & 0.1550 & 19.57 & 0.5543 \\
2 & 0.7 & 4 & 0.6155 & 0.2681 & 0.4569 & 18.82 & 0.5856 \\
3 & 0.5 & 5 & 0.9021 & 0.3033 & 0.6144 & 25.90 & 0.6787 \\
4 & 0.3 & 1 & 0.0468 & 0.0905 & 0.0437 & 9.12 & 1.1908 \\
5 & 0.1 & 0.5 & 0.0121 & 0.0476 & 0.0117 & 12.59 & 1.2232 \\
\hline
\end{tabular}


Case 2: Cost Function Table

\begin{tabular}{|c|c|c|c|c|c|c|c|}
\hline $\mathrm{n}$ & $\mathrm{P}$ & $\mathrm{t}$ & $H_{m}(t)=H_{o}(t)$ & $h_{m}(t)=h_{o}(t)$ & $Z_{m}(t)$ & $\mathrm{T}^{*}=|T|$ & $C_{2}(\mathrm{~T})$ \\
\hline 1 & 0.9 & 2 & 0.1730 & 0.1640 & 0.1550 & 23.40 & 0.8829 \\
\hline 2 & 0.7 & 4 & 0.6155 & 0.2681 & 0.4569 & 21.00 & 0.7706 \\
\hline 3 & 0.5 & 5 & 0.9021 & 0.3033 & 0.6144 & 27.07 & 0.7719 \\
\hline 4 & 0.3 & 1 & 0.0468 & 0.0905 & 0.0437 & 9.67 & 1.2934 \\
\hline 5 & 0.1 & 0.5 & 0.0121 & 0.0476 & 0.0117 & 12.60 & 1.2220 \\
\hline
\end{tabular}

Case 3: Cost Function Table

\begin{tabular}{ccccccc}
\hline $\mathrm{n}$ & $\mathrm{P}$ & $\mathrm{T}$ & $H_{m}(t)=H_{o}(t)$ & $h_{m}(t)=h_{o}(t)$ & $Z_{m}(t)$ & $C_{3}(\mathrm{~T})$ \\
\hline 1 & 0.9 & 2 & 0.1730 & 0.1640 & 0.1550 & 25.30 \\
2 & 0.7 & 4 & 0.6155 & 0.2681 & 0.4569 & 22.70 \\
3 & 0.5 & 5 & 0.9021 & 0.3033 & 0.6144 & 0.9428 \\
4 & 0.3 & 1 & 0.0468 & 0.0905 & 0.0437 & 1161 \\
5 & 0.1 & 0.5 & 0.0121 & 0.0476 & 0.0117 & 1.5706 \\
\hline
\end{tabular}

If the distribution is Weibull i.e. the extreme case of the exponential family, then the $p d f$ is given by

$f(t)=\gamma \beta t^{\beta-1} \mathrm{e}^{-\gamma \mathrm{t}^{\beta-1}}, t, \gamma, \beta>0$

Where $r(t)=\gamma \beta t^{\beta-1}, \bar{F}(t)=e^{-\gamma t^{\beta}}$,

$C_{1}(T)=\frac{n p \sum_{i=1}^{n} c_{1 i m}\left(\gamma T^{\beta}-\frac{\alpha \gamma \beta}{\beta+1} T^{\beta+1}\right) y_{m}}{t}+\frac{t n p \sum_{i=1}^{n} c_{1 i m}\left(\gamma \beta T^{\beta-1}-\alpha \gamma \beta T^{\beta}\right) x_{m}+(1-p) c_{i r} y_{m}+c_{B} y_{o}}{t}$

By setting $c^{\prime}{ }_{1}(t)=0$ we have

$\mathrm{T}^{*}=|T|=\sqrt[\beta+1]{\frac{\left(-(1-p) c_{1 r} y_{m}-c_{B} y_{o}\right)\left(\gamma y_{m}-t \alpha \gamma \beta x_{m}\right)}{n p \sum_{i=1}^{n} c_{1 i m}\left(\frac{\gamma(\beta+1)-\alpha \gamma \beta}{\beta+1} y_{m}+t(\gamma \beta-\alpha \gamma \beta) x_{m}\right)}}$

Also

$C_{2}(T)=\frac{n p \sum_{i=1}^{n} c_{1 i m}\left(\gamma T^{\beta}-\frac{\alpha \gamma \beta}{\beta+1} T^{\beta+1}\right) y_{m}+\operatorname{tnp} \sum_{i=1}^{n} c_{1 i m}\left(\gamma \beta T^{\beta-1}-\alpha \gamma \beta T^{\beta}\right) x_{m}+c_{p} y_{o}+c_{B} y_{o}}{t}$

By setting $c^{\prime}{ }_{2}(t)=0$ we have

$\mathrm{T}^{*}=|T|=\sqrt[\beta+1]{\frac{\left(-(1-p) c_{p} y_{o}-c_{B} y_{o}\right)\left(\gamma y_{m}-t \alpha \gamma \beta x_{m}\right)}{n p \sum_{i=1}^{n} c_{1 i m}\left(\frac{\gamma(\beta+1)-\alpha \gamma \beta}{\beta+1} y_{m}+t(\gamma \beta-\alpha \gamma \beta) x_{m}\right)}}$

Also,

$C_{3}(T)=\frac{n p \sum_{i=1}^{n} c_{1 i m}\left(\gamma T^{\beta}-\frac{\alpha \gamma \beta}{\beta+1} T^{\beta+1}\right) y_{m}+\operatorname{tnp} \sum_{i=1}^{n} c_{1 i m}\left(\gamma \beta T^{\beta-1}-\alpha \gamma \beta T^{\beta}\right) x_{m}+c_{c} y_{o}+c_{B} y_{o}}{t}$

By setting $c^{\prime}{ }_{3}(t)=0$ we have

$\mathrm{T}^{*}=|T|=\sqrt[\beta+1]{\frac{\left(-(1-p) c_{p} y_{o}-c_{B} y_{o}\right)\left(\gamma y_{m}-t \alpha \gamma \beta x_{m}\right)}{n p \sum_{i=1}^{n} c_{1 i m}\left(\frac{\gamma(\beta+1)-\alpha \gamma \beta}{\beta+1} y_{m}+t(\gamma \beta-\alpha \gamma \beta) x_{m}\right)}}$

\section{Numerical example}

Assume that the problem follows a Weibull distribution such that

$f(t)=\gamma \beta t^{\beta-1} e^{-\gamma t^{\beta-1}}, \gamma, \beta>0$

Assuming that $\gamma=100$ and $\beta=2$ then we can write

$f(t)=200 t e^{-100 t}$

We also assume the following values for the following parameters

$c_{11 \mathrm{~m}}=2.5, c_{12 \mathrm{~m}}=2.8, c_{13 \mathrm{~m}}=3.1, c_{14 \mathrm{~m}}=3.4, c_{15 \mathrm{~m}}=3.7$

$c_{1 r}=10.6, c_{p}=9.5, c_{c}=15.5, c_{B}=11.5, \alpha=0.02, \rho=0.1$

Calculating all the unknowns involved we have the following tables

Table I: When the Cost Function Is Case I

\begin{tabular}{ccccccc}
\hline $\mathrm{n}$ & $\mathrm{p}$ & $\mathrm{t}$ & $y_{m}$ & $x_{m}$ & $|T|$ \\
\hline 1 & 0.9 & 2 & 533.3 & 266.7 & 9.89 & 242.5 \\
2 & 0.7 & 4 & 2132.8 & 1066.7 & 9.25 \\
3 & 0.5 & 5 & 3332.5 & 1666.6 & 8.79 & 1252.1 \\
4 & 0.3 & 1 & 133.3 & 66.67 & 2897.3 & 20.2 \\
5 & 0.1 & 0.5 & 33.33 & 16.67 & 3.93 \\
\hline
\end{tabular}


Table II: When the Cost Function Is Case II

\begin{tabular}{|c|c|c|c|c|c|c|}
\hline $\mathrm{n}$ & $\mathrm{p}$ & $\mathrm{t}$ & $y_{m}$ & $x_{m}$ & $|T|$ & $C_{2}(t)$ \\
\hline 1 & 0.9 & 2 & 533.3 & 266.7 & 9.87 & 120.2 \\
\hline 2 & 0.7 & 4 & 2132.8 & 1066.7 & 9.19 & 1255.3 \\
\hline 3 & 0.5 & 5 & 3332.5 & 1666.6 & 8.69 & 2806.1 \\
\hline 4 & 0.3 & 1 & 133.3 & 66.67 & 4.40 & 17.19 \\
\hline 5 & 0.1 & 0.5 & 33.33 & 16.67 & 3.87 & 2.32 \\
\hline \multicolumn{7}{|c|}{ Table III: When the Cost Function Is Case III } \\
\hline $\mathrm{n}$ & $\mathrm{p}$ & $\mathrm{t}$ & $y_{m}$ & $x_{m}$ & $|T|$ & $C_{3}(T)$ \\
\hline 1 & 0.9 & 2 & 533.3 & 266.7 & 10.03 & 126.09 \\
\hline 2 & 0.7 & 4 & 2132.8 & 1066.7 & 9.55 & 1407.4 \\
\hline 3 & 0.5 & 5 & 3332.5 & 1666.6 & 9.20 & 3326.4 \\
\hline 4 & 0.3 & 1 & 133.3 & 66.67 & 4.72 & 23.7 \\
\hline 5 & 0.1 & 0.5 & 33.33 & 16.67 & 4.19 & 2.75 \\
\hline
\end{tabular}

\section{Conclusion}

In this research work, a system that has no directly predictable arrangement is considered (that is, the system arrangement is not parallel, seriesor parallel-series). The system is group into two where the first group consist of system components that at their failure they are repairable and replaceable and the second group consist of components that are non-repairable but replaceable at their failure. Two types of failures are considered, as type y failure is for group A and type $\mathrm{z}$ failure is for group B. Therefore the system is replace at nth type $\mathrm{y}$ failure or at first type zfailure or when the total operating time reaches age $\mathrm{T}$. the possible cost of maintenance is derived and optimum replacement period is obtained using both the exponential and Weibull distribution, numerical example is given to make the empirical realization of the model.

\section{References}

[1] Chien, Y.H., Sheu, S.H., Zhang, Z.G. and Ernie, L."An extended optimal replacement model of systems subject to shocks" European journal of operational research, vol. 175pp399-412, 2006.

[2] Sheu, S.H., Griffith, W.S. and Nakagawa, T., "Extended optimal replacement model with random minimal repair costs", European journal of operational research, vol. 85 pp636-649, 1995 http://dx.doi.org/10.1016/0377-2217 (93) E0364-4.

[3] Aven T. and Castro, I.T., "A minimal repair replacement model with two types of failure and a safety constraint", European journal of operational research, vol. 188 pp506-515, 2008 http://dx.doi.org/10.1016/j.ejor.2007.04.038.

[4] Chien Y.H. and Sheu, S.H., "Extended optimal age replacement policy with minimal repair of a system subject to shocks", European journal of operational research, vol.174 pp169-181, 2006 http://dx.doi.org/10.1016/j.ejor.2005.01.032.

[5] Yakasai, B.M., "A cast-off replacement policy for a component demanded by two parallel units", Journal operational research society, vol. 52pp716-723, 2001

[6] Castro I.T. and Sanjuan, E.L., "An optimal repair policy for systems with limited number of repairs", European journal of operational research, vol. 198 pp88-96, 2007.

[7] Philip A.S and Muhammad D, "Block replacement policies for a two component system with failure dependence", Naval research logistic, vol. 50 pp70-87, 2003.

[8] Nakagawa T., "Replacement problem of a parallel system in random environment", journal of applied probability, vol. 16 pp203-205, 1979.

[9] Zequeira R. I. and Berenguer C., "Periodic imperfect preventive maintenance with two categories of competing failure modes", Reliability engineering and system safety, vol. $91 \mathrm{pp} 460$ - 468, 2006 http://dx.doi.org/10.1016/j.ress.2005.03.009.

[10] Wang H., "A survey of maintenance policies of deteriorating systems", European journal of operation research, vol. 139 pp469 -489, 2002.

[11] Aven T and Jensen U.,"A general minimal repair model", journal of applied probability, vol. 37, pp187 - 197, 2000.

[12] Barlow R. E. and Proschan F., "Mathematical theory of reliability", Wiley, New York, 1965.

[13] Cox D. R., "Renewal theory", Chapman and hall, New York, 1982. 